\title{
CORRECTION
}

\section{Author Correction: Salvianolic acid A prevented cerebrovascular endothelial injury caused by acute ischemic stroke through inhibiting the Src signaling pathway}

Cheng-di Liu ${ }^{1}$, Nan-nan Liu ${ }^{1}, 2$, Sen Zhang ${ }^{1}$, Guo-dong Ma ${ }^{1}$, Hai-guang Yang ${ }^{1}$, Ling-lei Kong ${ }^{1}$ and Guan-hua Du ${ }^{1}$ Acta Pharmacologica Sinica (2021) 42:1014; https://doi.org/10.1038/s41401-020-00607-y

Correction to: Acta Pharmacol Sin https://doi.org/10.1038/s41401020-00568-2, published online 10 Dec 2020

The authors apologize that the dosage of SAA is incorrectly written as $100 \mathrm{mg} / \mathrm{kg}$ in Fig. $1 \mathrm{~b}$ in the online version. The correct dose is $10 \mathrm{mg} / \mathrm{kg}$ and we have revised Fig. 1. The revisions do not affect any results and conclusions of the current work. The authors apologize for any inconvenience caused to the journal and readers.

Figure 1 is revised<smiles>O=C(/C=C/c1ccc(O)c(O)c1/C=C/c1ccc(O)c(O)c1)O[C@@H](Cc1ccc(O)c(O)c1)C(=O)O</smiles>

Salvianolic acid $\mathrm{A}$ b
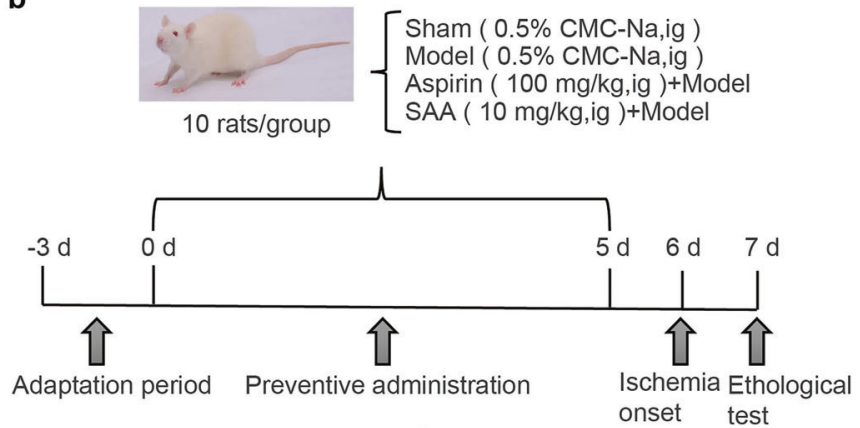

Fig. 1 Chemical structure of SAA and a schematic diagram of the experimental protocols. a Chemical structure of SAA. b Schematic diagram of the experimental protocols.

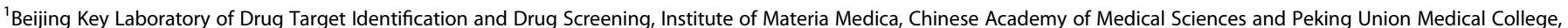
Beijing 100050, China and ${ }^{2}$ Guangdong Pharmaceutical University, Guangzhou 510006, China

Correspondence: Ling-lei Kong (konglinglei@imm.ac.cn) or Guan-hua Du (dugh@imm.ac.cn)

Published online: 29 January 2021 